Published in final edited form as:

Hum Pathol. 2018 February ; 72: 35-44. doi:10.1016/j.humpath.2017.08.006.

\title{
Carcinoma of the Urethra
}

\author{
Miao Zhang, MD $^{\star}$, Adebowale J. Adeniran, MD $\ddagger$, Raghunandan Vikram, $\mathbf{M D}^{\dagger}$, Pheroze \\ Tamboli, MD*, Curtis Pettaway, MD\#, Jolanta Bondaruk, PhD*, Jinsong Liu, MD, PhD*, Keith \\ Baggerly, $\mathrm{PhD}^{ \pm}$, and Bogdan Czerniak, MD, PhD* \\ "Department of Pathology, The University of Texas M.D. Anderson Cancer Center, Houston, TX, \\ United States. \\ tDepartment of Radiology, The University of Texas M.D. Anderson Cancer Center, Houston, TX, \\ United States. \\ \#Department of Urology, The University of Texas M.D. Anderson Cancer Center, Houston, TX, \\ United States. \\ ${ }^{ \pm}$Department Bioinformatics and Computational Biology, The University of Texas M.D. Anderson \\ Cancer Center, Houston, TX, United States. \\ ‡Department of Pathology, Yale University, New Haven, CT, United States.
}

\section{SUMMARY}

Primary carcinomas of the urethra are rare and poorly understood lesions, hence their clinical and pathologic spectrum is not completely defined. We analyzed a series of 130 primary urethral tumors and classified 106 of them as primary urethral carcinomas. The age at diagnosis of patients with primary urethral carcinomas ranged from 42-97 years (mean: 69.4 yrs; median: 70 yrs). There were 73 males and 33 female patients with a ratio of 2.2:1. In male patients the tumors most frequently developed in the bulbous-membranous segment of the urethra. In female patients the entire length of the urethra was typically involved. Microscopically, they were poorly differentiated carcinoma with hybrid squamous and urothelial features and developed from precursor intraepithelial conditions such as dysplasia and carcinoma in situ, which were frequently present in the adjacent urethral mucosa. High risk HPV infection could be documented in $31.6 \%$ of these tumors. Follow-up information was available for 95 patients. Twenty-three patients died of the disease with a mean and median survival of 39 and 21 months respectively. Urethral carcinomas are aggressive tumors with high propensity for regional and distant metastases with mean and median survival of 39 and 21 months respectively. Our observations have important implications for the management of patients with primary carcinoma of the urethra by defining them as a unique entity linked to HPV infection.

*Correspondence author. Department of Pathology, Unit 085, The University of Texas MD Anderson Cancer Center, 1515 Holcombe Boulevard, Houston, TX 77030; USA Tel. 713-794-1025; bczernia@ mdanderson.org (B. Czerniak).

Publisher's Disclaimer: This is a PDF file of an unedited manuscript that has been accepted for publication. As a service to our customers we are providing this early version of the manuscript. The manuscript will undergo copyediting, typesetting, and review of the resulting proof before it is published in its final citable form. Please note that during the production process errors may be discovered which could affect the content, and all legal disclaimers that apply to the journal pertain.

Conflict of Interest: The authors declare no conflict of interest. 


\section{Keywords}

Urethral carcinoma; HPV-related cancer

\section{INTRODUCTION}

Primary carcinomas of the urethra are rare and account for less than $1 \%$ of urinary tract malignancies(1-3). The histologic type, natural history, treatment and prognosis associated with these tumors are, to a large extent, determined by the segment of the urethra in which they develop(1). Because of the rarity of this entity, most cases in the literature represent single case reports or small series(4-12), hence their clinical and morphologic spectrum remains incompletely defined and their behavior is poorly understood.

Under the current World Health Organization-based scheme, carcinomas of the urethra are classified as urothelial, squamous or adenocarcinomas of either unspecified or enteric type occasionally exhibiting mucinous features with the remaining being extremely rare clear cell and adenoid cystic carcinomas(13). The tumors involving the proximal urethra, including those arising in the prostatic segment in males, are frequently secondary tumors of urothelial origins extending from the bladder(13). On the other hand, the tumors of the distal urethra, especially those involving the meatus, are frequently of squamous type and may represent secondary involvement by the extension from the lesions primary in the glans penis in males or vulva in females(14-16). These tumors were eliminated from the analysis and in this report we focused only on 130 primary tumors developing in the urethra. The analysis of this material identified 106 primary urethral carcinomas while the remaining 23 tumors were adenocarcinomas of various types and one case of adenoid cystic carcinoma.

Here, we propose a new entity that we define as a primary urethral carcinoma, which typically is poorly differentiated, and shows hybrid features of urothelial and squamous differentiation. This carcinoma is distinct from typical urothelial carcinomas developing in the bladder, and squamous carcinomas developing in the distal male urethra involving meatus or glans penis as well as those arising in the vulva of females. We believe this carcinoma evolves from intraepithelial precursor conditions in the urethral mucosa through the dysplasia/carcinoma in situ sequence.

\section{MATERIALS AND METHODS}

\section{Tissue Samples and Patient Data}

The study was approved by the MD Anderson Cancer Center Institutional Review Board and the pathology and clinical archival files were searched for primary urethral carcinomas of patients seen between January, 1984 and June, 2014. Pathology reports, hematoxylin and eosin slides and pertinent immunohistochemical studies were reviewed. Cases with secondary involvement of the urethra by urinary bladder carcinoma or squamous carcinoma from glans penis or vulva were excluded. Cases were reviewed by 3 expert genitourinary pathologists (M.Z, P.T and BC). Tumors were evaluated for gross appearance, size, grade, in-situ component, histological features, adjacent organ/structure involvement, and 
lymphovascular invasion. Clinical information, including patient age, gender, prior cancer history, cystoscopy findings and radiographic imaging data were analyzed and complemented with treatment information and the follow up data on the development of metastases and survival.

\section{Immunohistochemical Staining}

The following immunohistochemical studies were performed: KRT5/6 (DAKO, diluted 1:100), KRT7 (DAKO, diluted 1:100), KRT 20 (DAKO, diluted 1:40), P63 (Santa Cruz, diluted 1:1000), P16 (Ventana, diluted 1:3), thrombomodulin (DAKO, diluted 1:40), GATA3 (Leica Microsystems, diluted 1:200), and uroplakin III (Fitzgerald, diluted 1:40). The bound antibodies were visualized with biotinylated Dextran backbone polymer and DAB and counterstained with hematoxylin.

To perform RNA scope analysis for HPV we constructed tissue microarrays in which three $2 \mathrm{~mm}$ tissue blocks were used as representatives of each case to construct the mother block containing 19 cases of urethral carcinomas and 17 cases of penile squamous carcinomas as previously described(17). Penile squamous carcinomas were included in the microarrays as a reference control set. RNA scope analysis was carried out according to the manufacturer's recommendation (Advanced Cell Diagnostic). In brief, sections of tissue microarray after deparaffinization and dehydration were incubated with a mixture of seven probes for high risk HPV types 16, 18, 31, 33, 35, 52 and 58 referred to as HR7 and a bacterial gene dap $\beta$ probe with amplifiers. The hybridized probes were visualized with DAB Mixture for signal detection and the slides counter stained with hematoxylin.

\section{Statistical analysis}

The overall survival outcomes and in relation to gender, stage, and histologic grade were analyzed by using log-rank test and Kaplan Meier curves. The association among age, gender, stage, and histologic grade and survival were analyzed in a multivariate fashion using Cox proportional hazard model. For the purpose of survival analyses the tumors were classified according to their histologic grade as well-, moderately, and poorly differentiated corresponding to numerical grades $1-3$ and were dichotomized into Ta- $\mathrm{T}_{1}$ (superficial early invasive) versus $T_{2}$ and higher (deeply invasive) according to the WHO classification of tumors of the urinary system and the American Joint Committee on Cancer TNM staging system. $(18,19)$

\section{RESULTS}

The analysis of 130 cases classified as primary urethral tumors disclosed that 24 of them could be classified microscopically as tumors with specific features such as adenocarcinomas (23), and adenoid cystic carcinoma (1). The remaining 106 were poorly differentiated carcinomas with hybrid squamous and urothelial features, which we defined as primary carcinomas of the urethra, which are the focus of this report. The clinical and pathological features of these cases are summarized in Table 1. 


\section{Clinical Features}

Among the 106 cases of primary urethral carcinoma there were 73 men and 33 women (ratio 2.2:1). The patients' age at the time of diagnosis ranged from 42 to 97 years (mean: 69.4 years; median: 70 years). They presented with dysuria, decreased urinary stream, urinary retention and perineal pain. Urethroscopic/cystoscopic data available for 76 cases disclosed that 60 patients presented with a mass lesion while 15 cases showed endoscopic evidence of urethral narrowing. In the remaining case, no endoscopically detectable lesion could be identified and multiple mapping urethral biopsies were required to detect the tumor. The patients were typically treated with radical urethral resections, which depending on the tumor location and tumor stage, required additional cystectomy, or prostatectomy, or cystoprostatectomy in men; with pelvic exenteration in advanced cases. In cases with regional lymph node metastases, the surgery involved inguinal and pelvic lymph node dissections.

\section{Radiographic imaging}

Pre-surgical magnetic resonance imaging (MRI) data were available for 15 patients (11 males and 4 females) and the primary urethral tumors were visible on the MRIs in all patients. The tumors showed intermediate signal intensity on T2 weighted images (Fig. 1A, D and Supplementary Fig. S1A, D). Post contrast images typically showed low to moderate enhancement within the tumor. The membranous or bulbous urethra was involved in 6 of the male cases while the penile urethra was involved in the remaining 5 male patients. The tumor invaded the corpus spongiosum in 8 patients which was best documented on orthogonal plane images showing the breakdown of the low signal in the muscularis with contiguous changes in signal intensity in the adjacent corpus spongiosum on $\mathrm{T} 2$ weighted images (Supplementary Fig. S2A-C). In two of these patients the tumor was seen invading the surrounding Buck's fascia and the corpus cavernosum. In two female patients, the tumor involved the entire length of the urethra from the base of the urinary bladder to the urethral meatus (Supplementary Fig. S3A, B). One female patient had a tumor within the urethral diverticulum (Supplementary Fig. S4A-D) and the one remaining female patient had a tumor involving the distal urethra.

\section{Pathologic features}

Tumors were poorly circumscribed ranging in size from 0.5 to $6 \mathrm{~cm}$ (mean $3.7 \mathrm{~cm}$ ) with a white to yellow-tan appearance. After opening of the urethra some of the tumors appeared as fungating masses protruding to the urethral lumen with cobbled and papillary surface (Fig. 1B, C). Others were flat lesions which extensively infiltrated the surrounding tissue causing urethral stenosis (Supplementary Fig. S1B, C). The tumor masses infiltrated adjacent structures surrounding the urethra, the urogenital diaphragm, the periurethral portion of the corpus spongiosum, the corpora cavernosa, and in some cases, the prostate gland.

Histologically, primary urethral carcinomas were poorly differentiated with small to intermediate cells showing high nuclear to cytoplasmic ratio. The nuclei were ovoid or round in shape and were often vertically oriented at the periphery of cancer nests forming basaloid or palisading structures (Fig. 2A-D). They showed occasional nuclear overlap and nuclear groves. Most cases showed mixed morphologic features of squamous and urothelial 
differentiation. Squamous differentiation was evident by the frequent presence of foci of variable keratinization with occasional well differentiated areas showing intercellular bridges (Fig. 3A, B). Urothelial differentiation showed features of papillary architecture especially evident on the tumor surface with typical urothelium-like stratification in the overlying epithelial layer (Fig. 3C, D). In contrast to long, thin and branching papillary structures with narrow fibrovascular cores seen in conventional papillary urothelial carcinomas, the papillary structures of urethral carcinomas were short and irregular with broad fibrovascular cores. Areas representing a fusion of irregular papillary structures were also present. Brisk mitotic activity with atypical mitoses as well as foci of necrosis were constant characteristics of these tumors. In some cases, intraepithelial in-situ precursor lesions were present in the adjacent urethral mucosa (Fig. 4A-D). They represented a full spectrum of mild and moderate to severe dysplasia/carcinoma in-situ lesions.

Immunohistochemical stains performed on the selected cases of primary urethral carcinomas are summarized in Fig. 5A-D and Table 1. These tumors were positive for KRT5/6 (87.1\%), KRT7 (66.1\%), P63(80.6\%), P16(62.8\%) and throbomodulin (82.2\%) in the majority of cases. Expression of KRT20 could be detected in $9.6 \%$ of the cases while GATA3 was focally positive in $3.9 \%$ of the cases. Although primary urethral carcinomas microscopically exhibit urothelial features they do not express markers of terminal urothelial differentiation such has uroplakin. RNA scope analysis showed that 6 of 19 (31.6\%) urethral carcinomas were positive for high risk HPV while 4 of 17 (23.5\%) of penile squamous carcinomas were positive for the same set of high risk HPV probes (Fig. 5D inset).

\section{Treatment and Follow-up}

All patients were treated with surgery and some of them received additional chemo- and/or radiation therapy. Of 29 patients who received additional therapy, 15 were treated with chemoradiation, 10 received chemotherapy and 4 radiation therapy only. Follow up information was available for 95 patients ranging from 1 to 237 months (average of 38 months). Regional lymph node metastases developed in 27 patients with the inguinal lymph nodes being the most commonly affected site. Distant metastases developed in 16 patients with the lung and liver as the most frequent sites in 7 and 3 patients respectively. Twentythree patients died of the disease with a mean and median survival of 39 and 21 months respectively. There were 48 patients alive with 25 patients being alive with disease and 23 patients with no evidence of disease. The rest of the patients (24) were either dead of other or unknown causes.

Finally we addressed the issue of whether various clinical and pathological parameters including age, gender, stage, and histologic grade can be used to predict clinical behavior of primary urethral carcinomas. The overall Kaplan Meier survival analyses confirmed that urethral carcinomas were highly aggressive tumors with a 25\% 10 year survival rate. (Fig. 6A) The Kaplan Meier survival analyses in relation to histologic grade, stage, and gender showed that females had slightly better survival when compared to males and tumors of high histologic grade and high stage $\mathrm{T}_{2}$ and higher were more aggressive, however the differences were not statistically significant. (Fig. 6B-C) Similarly, the multivariate analyses showed that none of the clinical and pathological parameters can be used to reliably predict survival 
confirming that urethral carcinomas should be considered as being universally highly aggressive even in the early superficially invasive stage.

\section{DISCUSSION}

The analysis of our data indicate that a small fraction of the primary tumors arising in the urethra can be microscopically classified as tumors with specific features such as adenocarcinomas including clear cell adenocarcinomas, as well as extremely rare adenoid cystic carcinomas $(1,13,20-22)$. The vast majority of the tumors arising in the epithelial membrane of the urethra are the so-called primary urethral carcinomas which are poorly differentiated and exhibit hybrid features of squamous and urothelial differentiation(22). They are, however, distinct from conventional urothelial carcinomas frequently arising in the bladder as well as squamous carcinomas arising in the glans penis or vulva. The presence of dysplasia/carcinoma in situ-like lesions in the adjacent urethral mucosa which can be documented in some of the cases, further confirms their urethral origin.

Immunohistochemically, these tumors are frequently positive for such epithelial markers such as KRT5/6, KRT7, P63 and P16. They also express thrombomodulin, which is frequently expressed in urothelial carcinomas but are negative for GATA3 in the vast majority of cases. They are also negative for markers of terminal urothelial differentiation such as uroplakin. Squamous differentiation is frequently present on this tumor and over half are positive for P16. They appear to develop in association with HPV infection and high risk HPV can be detected in $31.6 \%$ of the cases. In several organ sites, P16 positivity is used as a surrogate for HPV infection and the strong positivity in more than $70 \%$ of the tumor tissue is considered indicative of HPV infection. (23-28) In the vast majority of our cases, P16 was strongly positive in more than $70 \%$ of the tumor tissue suggesting that HPV infection may be involved in nearly $50 \%$ of urethral carcinomas. Unfortunately, there was a minimal overlap between the tumors which were stained for P16 and those for which the HPV status was analyzed by the RNA-scope analysis. Hence the correlation between P16 positivity and the HPV status in reference to urethral carcinomas remains uncertain. Overall, these tumors are similar to the so called basaloid variant of penile squamous carcinoma and have overlapping features of a newly described basal subtype of bladder cancer(13, 29).

Recent genomic studies of bladder cancer have disclosed that it can be separated into two major groups referred to as luminal and basal in which the tumors recapitulate the expression profile of the less differentiated basal layer of the urothelium or its more differentiated intermediate to luminal layers(29-31). The luminal tumors develop via the papillary pathway and express CK18, CK20, and uroplakin, as well as GATA3 and PPAR $\lambda$ transcription factors characteristic of intermediate and luminal terminal urothelial differentiation(32). Practically all superficial papillary urothelial carcinomas are of luminal type and approximately $50 \%$ of invasive urothelial tumors belong to this category. The basal tumors are typically negative for GATA 3 and PPAR $\lambda$ and show expressions of CK5/6 and CK14 as well as p63 transcription factor. Approximately half of invasive bladder tumors are of basal molecular subtype and are microscopically poorly differentiated with palisading tumor nuclei at the periphery of tumor nests as well as enrichment for squamous differentiation. Both the immunophenotype as well as the microscopic features of the basal 
molecular subtypes of bladder cancer may overlap with primary urethral carcinomas but, in contrast, urothelial tumors are practically never positive for high risk HPV.

Primary urethral carcinomas are distinctively different microscopically from the majority of squamous carcinomas developing in glans penis which are moderately to well differentiated squamous tumors with frequent verrucous features $(13,33)$. However, a unique poorly differentiated subset of penile cancer referred to as basaloid carcinoma with high incidents of high risk HPV infections is microscopically similar to primary urethral carcinoma(13, 33). The distinction should be based on the unique location of the tumor and its association with urethral epithelial precursor lesions.

The proximal urethra in males comprises the prostatic and membranous segments while the distal urethra consists of the bulbous and penile parts(34). It appears that the tumors developing in various segments of the urethra, especially those in males, may have distinct pathogenesis representing different microscopic categories $(35,36)$. The tumors developing in the proximal prostatic urethra may have pathogenesis overlapping with urothelial carcinomas. On the other hand, the lesions of the very distal urethral segment, near the navicular fossa and urethral meatus may overlap with squamous carcinoma of the glans penis in males and the vulva in females. The exact anatomic location of the tumor was not always available from our records but for those tumors with available data, it appears that the majority of primary urethral carcinomas in males develop in the bulbous and membranous segments of the urethra. The exact anatomic location in female patients was less evident as the vast majority of cases involve the entire length of the urethra but there was a subset of female patients who have tumors involving the distal segment of the urethra.

MRI is a helpful tool in aiding the diagnosis and it is used with increasing frequency in the pre-treatment staging of patients with carcinoma of the urethra(10,37, 38). Urethral tumors in both male and female patients are best seen on sagittal images which provide useful information on the epicenter of the lesion in relation to the urethral segment and adjacent anatomic structures. Urethral tumors show low signal intensity on T1 weighted images and intermediate signal intensity higher than the skeletal muscle on T2 weighted images. The corpus spongiosum and corpora cavernosa in male patients return a high signal on $\mathrm{T} 2$ weighted images in contrast to the tumor which shows low to intermediate signal intensity. The muscularis layer of the urethra in male patients is seen as a low signal intensity structure which is interrupted by tumor if there is invasion of the surrounding corpus spongiosum. Differential enhancement on post contrast MRI provides additional information in local staging. In female patients, MRI of the urethra in an axial plane shows alternating high and low signal rings giving an overall targetoid appearance on $\mathrm{T} 2$ weighted images which correspond to the concentric layers of urethral mucosa, sub-mucosa, smooth muscle layer and surrounding skeletal muscles providing an opportunity to assess the epicenter of the lesion and the involvement of the adjacent structures. The MRI features in both male and female patients provide important information for the origin of a tumor as well as the involvement of adjacent structures which are helpful for therapy planning.

The family of HPV viruses, especially those referred to as high risk such as HPV 16 and HPV 18, are implicated in the development of multiple human malignancies(23). The 
prototypic human malignancy linked to HPV infection is the female uterine cervix carcinoma as well as carcinomas developing in the vagina and vulva(24). The prototypic male cancer associated with HPV infection is a penile cancer typically developing in the glans penis $(25,26,33)$. More recently, a subset of oropharyngeal cancer was also linked to the HPV infection $(27,28)$. The identification of primary urethral carcinoma as a distinct entity further expands the list of human cancers linked to the HPV infection which in our series can be identified in $32 \%$ of the cases.

Primary urethral carcinomas are aggressive tumors with a high propensity for local invasive growth as well as regional and distal metastases. Invasion of the lyphovascular spaces of the corpora and of the periurethral tissue is common. The anatomic location of the tumor may be associated with a unique spread as lyphatic channels from the distal urethra drain into the inguinal lymph nodes, whereas those from the proximal urethra drain into the deep pelvic and hypogastric lymph nodes. The tumors of the bulbous and membranous urethra have a tendency to invade the deep structures of the perineum and metastasize to the iliac and hypogastric lymph nodes. In contrast, tumors arising in the distal urethra preferentially metastasize to the inguinal lymph nodes. In our study, 26 cases showed evidence of lymph node metastases with the inguinal lymph nodes being the most frequently affected. The most common distant metastatic sites in our series were the lung and liver. Our follow-up data and survival analyses clearly indicated the aggressive nature of the primary urethral carcinomas with mean and median survival of 39 and 21 months respectively.

\section{CONCLUSION}

We propose a new entity of a primary urethral carcinoma most frequently arising in the bulbous and membranous male urethra from precursor urothelial conditions such as dysplasia and carcinoma in situ. These tumors are poorly differentiated carcinomas with basaloid features and hybrid squamous as well as urothelial differentiation. They develop in association with HPV infection and are highly aggressive clinically with frequent regional lymph node and distant organ metastatic spread.

\section{Supplementary Material}

Refer to Web version on PubMed Central for supplementary material.

\section{Acknowledgments}

Funding

This study was supported in part by the GU Bladder SPORE grant from NIH (P50 CA91846/Project 1 and Core C) to $\mathrm{BC}$.

\section{References}

1. Melicow MM, Roberts TW. Pathology and natural history of urethral tumors in males. Review of 142 cases. Urology. 1978; 11:83-89. [PubMed: 622771]

2. Amin MB, Young RH. Primary carcinomas of the urethra. Semin Diagn Pathol. 1997; 14:147-160. [PubMed: 9179975] 
3. Ampil FL. Primary malignant neoplasm of the female urethra. Obstet Gynecol. 1985; 66:799-804. [PubMed: 3934611]

4. Yvgenia R, Ben Meir D, Sibi J, Koren R. Mucinous adenocarcinoma of posterior urethra. Report of a case. Pathol Res Pract. 2005; 201:137-140. [PubMed: 15901135]

5. Zinman LN, Vanni AJ. Management of Proximal Primary Urethral Cancer: Should Multidisciplinary Therapy Be the Gold Standard? Urol Clin North Am. 2016; 43:505-513. [PubMed: 27717436]

6. Dayyani F, Hoffman K, Eifel P, Guo C, Vikram R, Pagliaro LC, Pettaway C. Management of advanced primary urethral carcinomas. BJU Int. 2014; 114:25-31. [PubMed: 24447439]

7. Tesluk H. Primary adenocarcinoma of female urethra associated with diverticula. Urology. 1981; 17:197-199. [PubMed: 6781117]

8. Suzuki K, Morita T, Tokue A. Primary signet ring cell carcinoma of female urethra. Int J Urol. 2001; 8:509-512. [PubMed: 11683973]

9. Gakis G, Witjes JA, Comperat E, Cowan NC, De Santis M, Lebret T, Ribal MJ, Sherif AM. European Association of U. EAU guidelines on primary urethral carcinoma. Eur Urol. 2013; 64:823-830. [PubMed: 23582479]

10. Grivas PD, Davenport M, Montie JE, Kunju LP, Feng F, Weizer AZ. Urethral cancer. Hematol Oncol Clin North Am. 2012; 26:1291-1314. [PubMed: 23116581]

11. Elkon D, Kim JA, Huddleston AL, Constable WC. Primary carcinoma of the female urethra. South Med J. 1980; 73:1439-1442. [PubMed: 7444505]

12. Benson RC Jr, Tunca JC, Buchler DA, Uehling DT. Primary carcinoma of the female urethra. Gynecol Oncol. 1982; 14:313-318. [PubMed: 7152364]

13. Moch H, Cubilla AL, Humphrey PA, Reuter VE, Ulbright TM. The 2016 WHO Classification of Tumours of the Urinary System and Male Genital Organs-Part A: Renal, Penile, and Testicular Tumours. Eur Urol. 2016; 70:93-105. [PubMed: 26935559]

14. Corbishley CM, Rajab RM, Watkin NA. Clinicopathological features of carcinoma of the distal penile urethra. Semin Diagn Pathol. 2015; 32:238-244. [PubMed: 25656527]

15. Gadducci A, Tana R, Barsotti C, Guerrieri ME, Genazzani AR. Clinicopathological and biological prognostic variables in squamous cell carcinoma of the vulva. Crit Rev Oncol Hematol. 2012; 83:71-83. [PubMed: 22015047]

16. Yap J, O'Neill D, Nagenthiran S, Dawson CW, Luesley DM. Current insights into the aetiology, pathobiology, and management of local disease recurrence in squamous cell carcinoma of the vulva. BJOG. 2017; 124:946-954. [PubMed: 28081287]

17. Wang H, Wang H, Zhang W, Fuller GN. Tissue microarrays: applications in neuropathology research, diagnosis, and education. Brain Pathol. 2002; 12:95-107. [PubMed: 11770905]

18. WHO Classification of Tumours of the Urinary System and Male Genital Organs. Lyons: World Health Organization; 2016.

19. AJCC Cancer Staging Manual. Springer International Publishing. 2017

20. Meis JM, Ayala AG, Johnson DE. Adenocarcinoma of the urethra in women. A clinicopathologic study. Cancer. 1987; 60:1038-1052. [PubMed: 3038294]

21. Zhang M, Pettaway C, Vikram R, Tamboli P. Adenoid cystic carcinoma of the urethra/Cowper's gland with concurrent high-grade prostatic adenocarcinoma: a detailed clinicopathologic case report and review of the literature. Hum Pathol. 2016; 58:138-144. [PubMed: 27554206]

22. Bracken RB, Johnson DE, Miller LS, Ayala AG, Gomez JJ, Rutledge F. Primary carcinoma of the female urethra. J Urol. 1976; 116:188-192. [PubMed: 950701]

23. Hellner K, Dorrell L. Recent advances in understanding and preventing human papillomavirusrelated disease. F1000Res. 2017:6.

24. Lees BF, Erickson BK, Huh WK. Cervical cancer screening: evidence behind the guidelines. Am J Obstet Gynecol. 2016; 214:438-443. [PubMed: 26519782]

25. Stratton KL, Culkin DJ. A Contemporary Review of HPV and Penile Cancer. Oncology (Williston Park). 2016; 30:245-249. [PubMed: 26984219]

26. Alemany L, Cubilla A, Halec G, Kasamatsu E, Quiros B, Masferrer E, Tous S, Lloveras B, Hernandez-Suarez G, Lonsdale R, Tinoco L, Alejo M, Alvarado-Cabrero I, Laco J, Guimera N, Poblet E, Lombardi LE, Bergeron C, Clavero O, Shin HR, Ferrera A, Felix A, Germar J, Mandys 
V, Clavel C, Tzardi M, Pons LE, Wain V, Cruz E, Molina C, Mota JD, Jach R, Velasco J, Carrilho C, Lopez-Revilla R, Goodman MT, Quint WG, Castellsague X, Bravo I, Pawlita M, Munoz N, Bosch FX, de Sanjose S. group HVs. Role of Human Papillomavirus in Penile Carcinomas Worldwide. Eur Urol. 2016; 69:953-961. [PubMed: 26762611]

27. Udager AM, McHugh JB. Human Papillomavirus-Associated Neoplasms of the Head and Neck. Surg Pathol Clin. 2017; 10:35-55. [PubMed: 28153135]

28. Linxweiler M, Bochen F, Wemmert S, Lerner C, Hasenfus A, Bohle RM, Al-Kadah B, Takacs ZF, Smola S, Schick B. Combination of p16(INK4a) /Ki67 immunocytology and HPV polymerase chain reaction for the noninvasive analysis of HPV involvement in head and neck cancer. Cancer Cytopathol. 2015; 123:219-229. [PubMed: 25557267]

29. Choi W, Porten S, Kim S, Willis D, Plimack ER, Hoffman-Censits J, Roth B, Cheng T, Tran M, Lee IL, Melquist J, Bondaruk J, Majewski T, Zhang S, Pretzsch S, Baggerly K, Siefker-Radtke A, Czerniak B, Dinney CP, McConkey DJ. Identification of distinct basal and luminal subtypes of muscle-invasive bladder cancer with different sensitivities to frontline chemotherapy. Cancer Cell. 2014; 25:152-165. [PubMed: 24525232]

30. Choi W, Czerniak B, Ochoa A, Su X, Siefker-Radtke A, Dinney C, McConkey DJ. Intrinsic basal and luminal subtypes of muscle-invasive bladder cancer. Nat Rev Urol. 2014; 11:400-410. [PubMed: 24960601]

31. Czerniak B, Dinney C, McConkey D. Origins of Bladder Cancer. Annu Rev Pathol. 2016; 11:149_ 174. [PubMed: 26907529]

32. Dadhania V, Zhang M, Zhang L, Bondaruk J, Majewski T, Siefker-Radtke A, Guo CC, Dinney C, Cogdell DE, Zhang S, Lee S, Lee JG, Weinstein JN, Baggerly K, McConkey D, Czerniak B. MetaAnalysis of the Luminal and Basal Subtypes of Bladder Cancer and the Identification of Signature Immunohistochemical Markers for Clinical Use. EBioMedicine. 2016; 12:105-117. [PubMed: 27612592]

33. Sanchez DF, Canete S, Fernandez-Nestosa MJ, Lezcano C, Rodriguez I, Barreto J, AlvaradoCabrero I, Cubilla AL. HPV- and non-HPV-related subtypes of penile squamous cell carcinoma (SCC): Morphological features and differential diagnosis according to the new WHO classification (2015). Semin Diagn Pathol. 2015; 32:198-221. [PubMed: 25701382]

34. Pechriggl EJ, Bitsche M, Blumer MJ, Fritsch H. The male urethra: spatiotemporal distribution of molecular markers during early development. Ann Anat. 2013; 195:260-271. [PubMed: 23433589]

35. Faraj SF, Gonzalez-Roibon N, Munari E, Sharma R, Burnett AL, Cubilla AL, Netto GJ, Chaux A. Strong association of insulin-like growth factor 1 receptor expression with histologic grade, subtype, and HPV status in penile squamous cell carcinomas: a tissue microarray study of 112 cases. Virchows Arch. 2017

36. Chaux A, Munari E, Cubilla AL, Hicks J, Lecksell K, Burnett AL, Netto GJ. Immunohistochemical expression of the mammalian target of rapamycin pathway in penile squamous cell carcinomas: a tissue microarray study of 112 cases. Histopathology. 2014; 64:863871. [PubMed: 24279699]

37. Kawashima A, Sandler CM, Wasserman NF, LeRoy AJ, King BF Jr, Goldman SM. Imaging of urethral disease: a pictorial review. Radiographics. 2004; 24(Suppl 1):S195-216. [PubMed: $15486241]$

38. Stewart SB, Leder RA, Inman BA. Imaging tumors of the penis and urethra. Urol Clin North Am. 2010; 37:353-367. [PubMed: 20674692] 


\section{HIGHLIGHTS}

- Carcinomas of the urethra are rare and their full spectrum is not well defined.

- Urethral carcinoma has squamous and urothelial features and is associated with HPV infection.

- Urethral carcinoma is aggressive with a propensity for lymph node and distant organ metastases.

- This report is important for the management of patients with carcinoma of the urethra. 

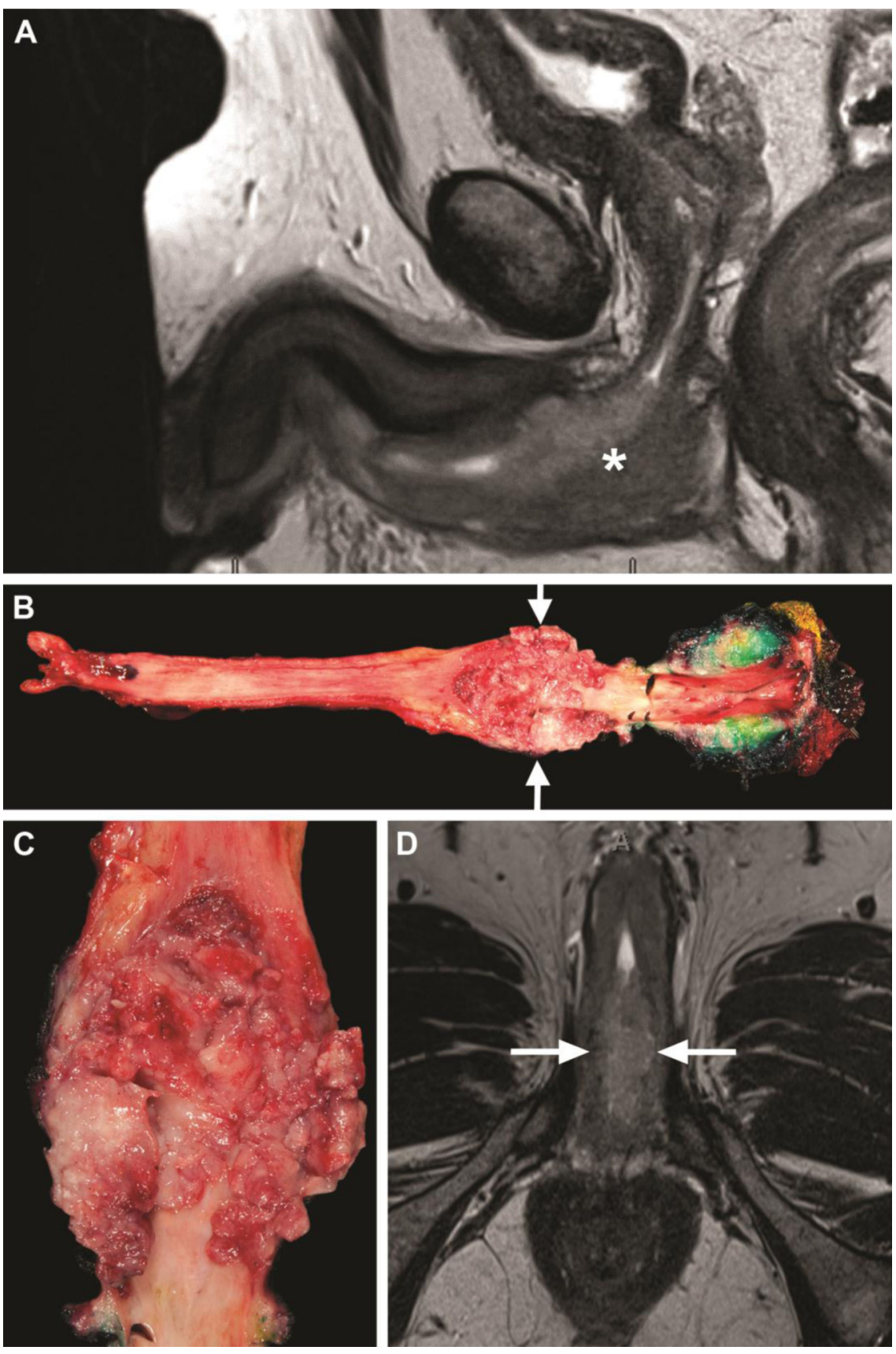

Fig. 1. Radiographic imaging and gross morphology of carcinoma developing in the male urethra (A) Sagittal MRI T2 weighted image of the pelvis showing a mass with intermediate signal intensity involving the bulbous urethra (asterix). (B) Total urethral and prostate resection specimen showing a fungating mass in the bulbous urethra (arrows). (C) Higher magnification of $\mathbf{B}$ showing the irregular cobbled and partially papillary surface of the fungating tumor in the bulbous segment of the urethra. (D) The axial MRI image at the level of the base of the penis showing the involvement of both corpora cavernosa (arrows). 

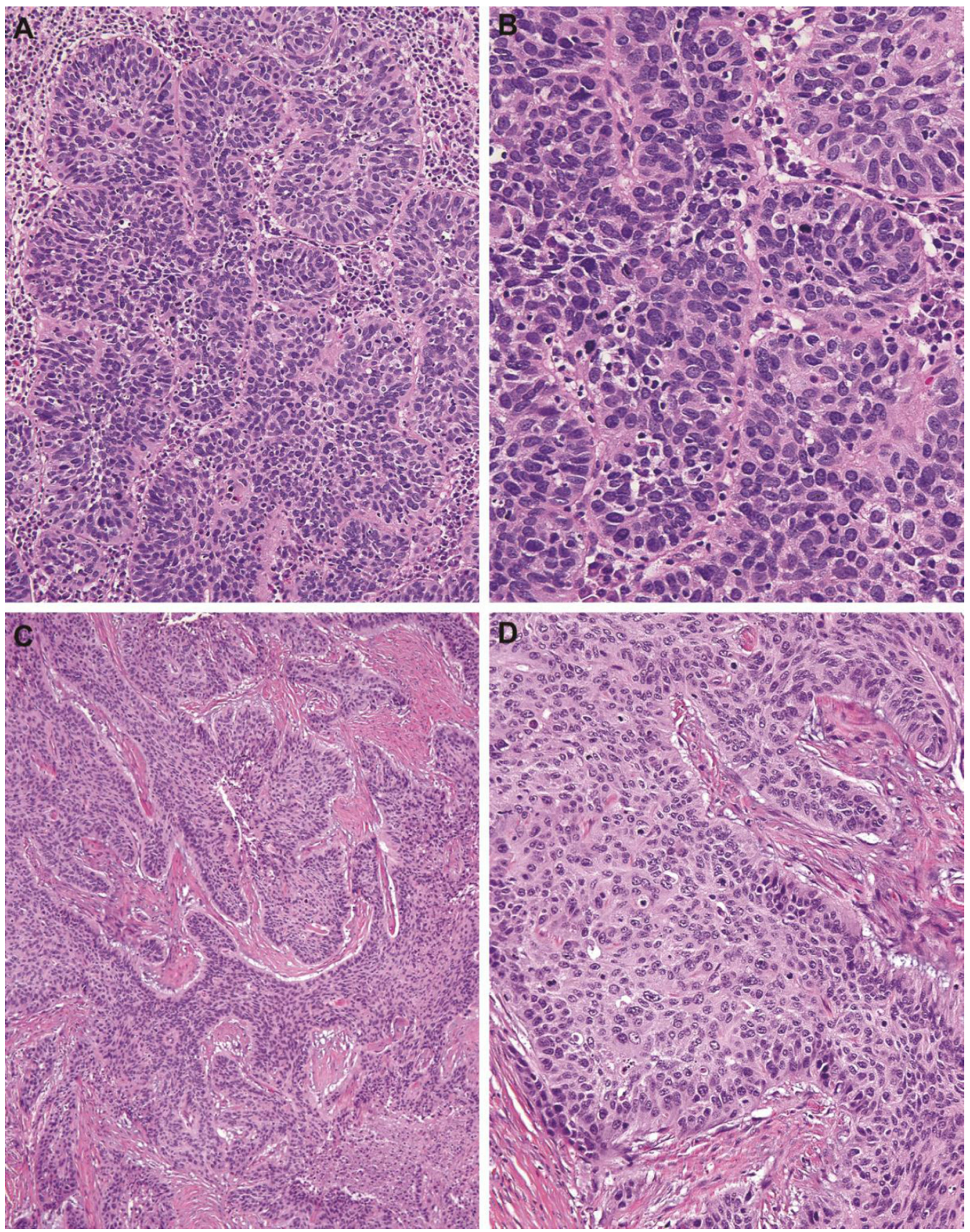

Fig. 2. Microscopic images of poorly differentiated urethral carcinoma with basaloid features (A and B) Intermediate and high power views of poorly differentiated tumor cells infiltrating stroma with a somewhat palisading arrangement of cells at the periphery of the tumor nests referred to as basaloid features. (C and D) Intermediate and high power views of poorly differentiated tumor cells infiltrating stroma and showing a palisading arrangement of tumor cells at the periphery of the tumor nests. (A-D hematoxylin and eosin; $\mathbf{A}$ and $\mathbf{D}$, $\times 200 ; \mathbf{B}, \times 400 ; \mathbf{C}, \times 100$ ) 

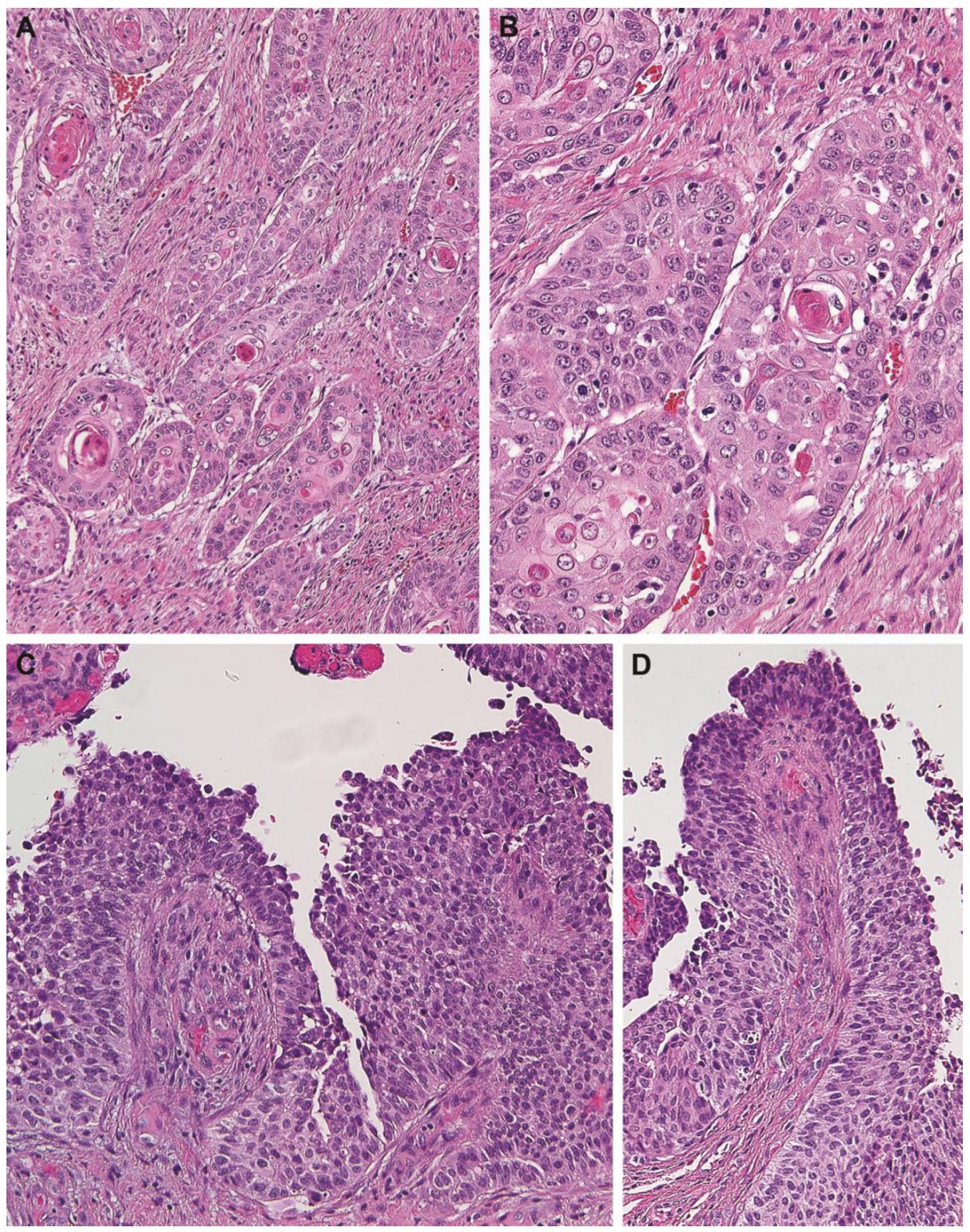

Fig. 3. Microscopic images of urethral carcinoma showing squamous and urothelial differentiation

(A and B) Irregular clusters of tumor cells showing features of squamous differentiation. (C and D) Papillary configuration of the tumor surface showing urothelium-like stratification in the epithelial layer. Note that in contrast to conventional urothelial carcinomas, these are irregular, short papillary structures with broad fibrovascular cores. (A-D: hematoxylin and eosin; A, ×200; B, ×400; C and D, $\times 100)$ 

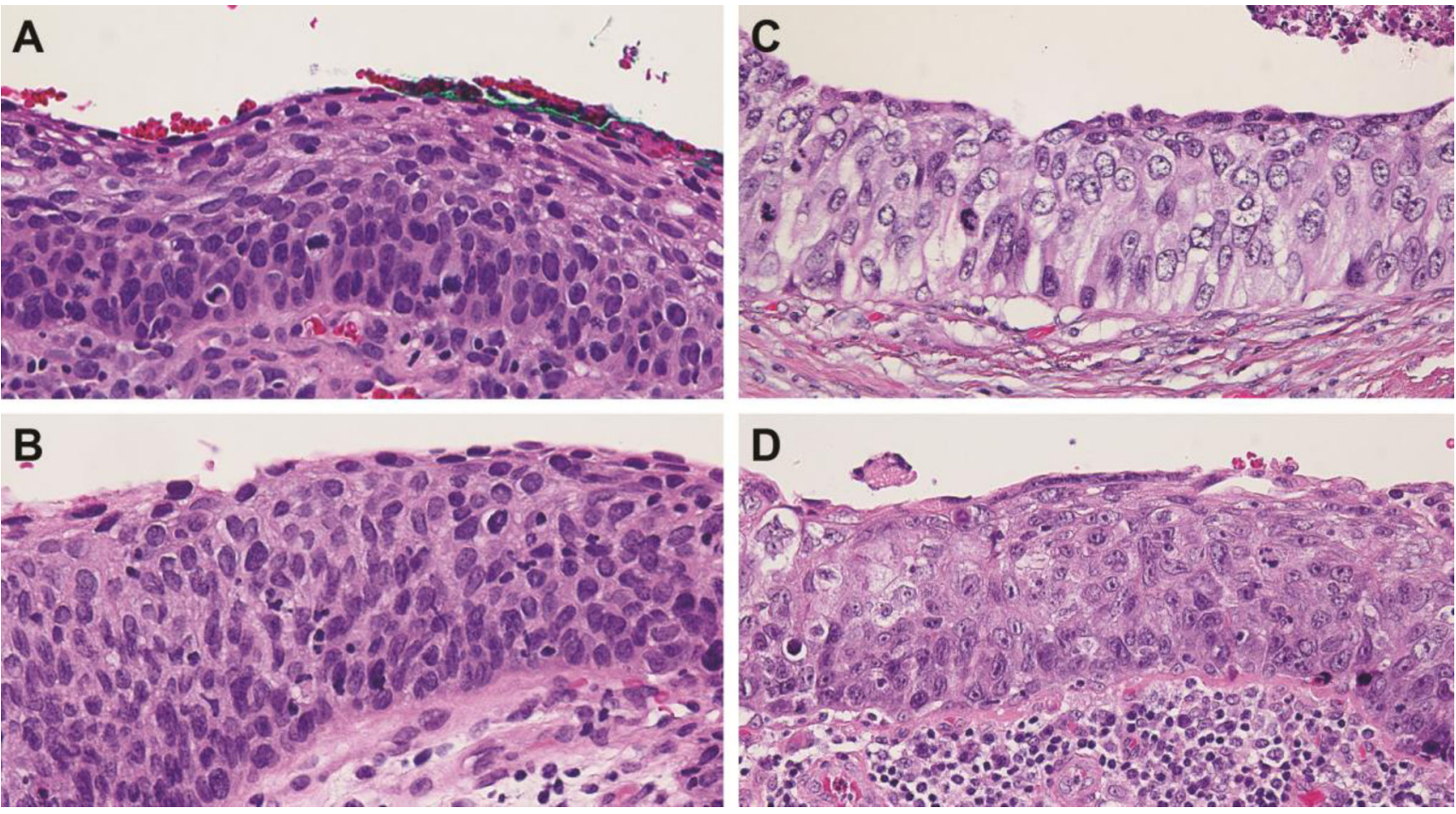

Fig. 4. Microscopic images of precursor intraepithelial lesions in the urethral mucosa adjacent to invasive cancer

(A and B) Proliferation of atypical epithelial cells with high N/C ratio and brisk mitotic activity showing some degree of differentiation and stratification towards the luminal layer of epithelium consistent with severe dysplasia. (C and D) Proliferation of atypical undifferentiated cells, with a high N/C ratio throughout the epithelium, which show brisk mitotic activity consistent with carcinoma in situ. (A-D: hematoxylin and eosin; A-D, $\times 400)$ 

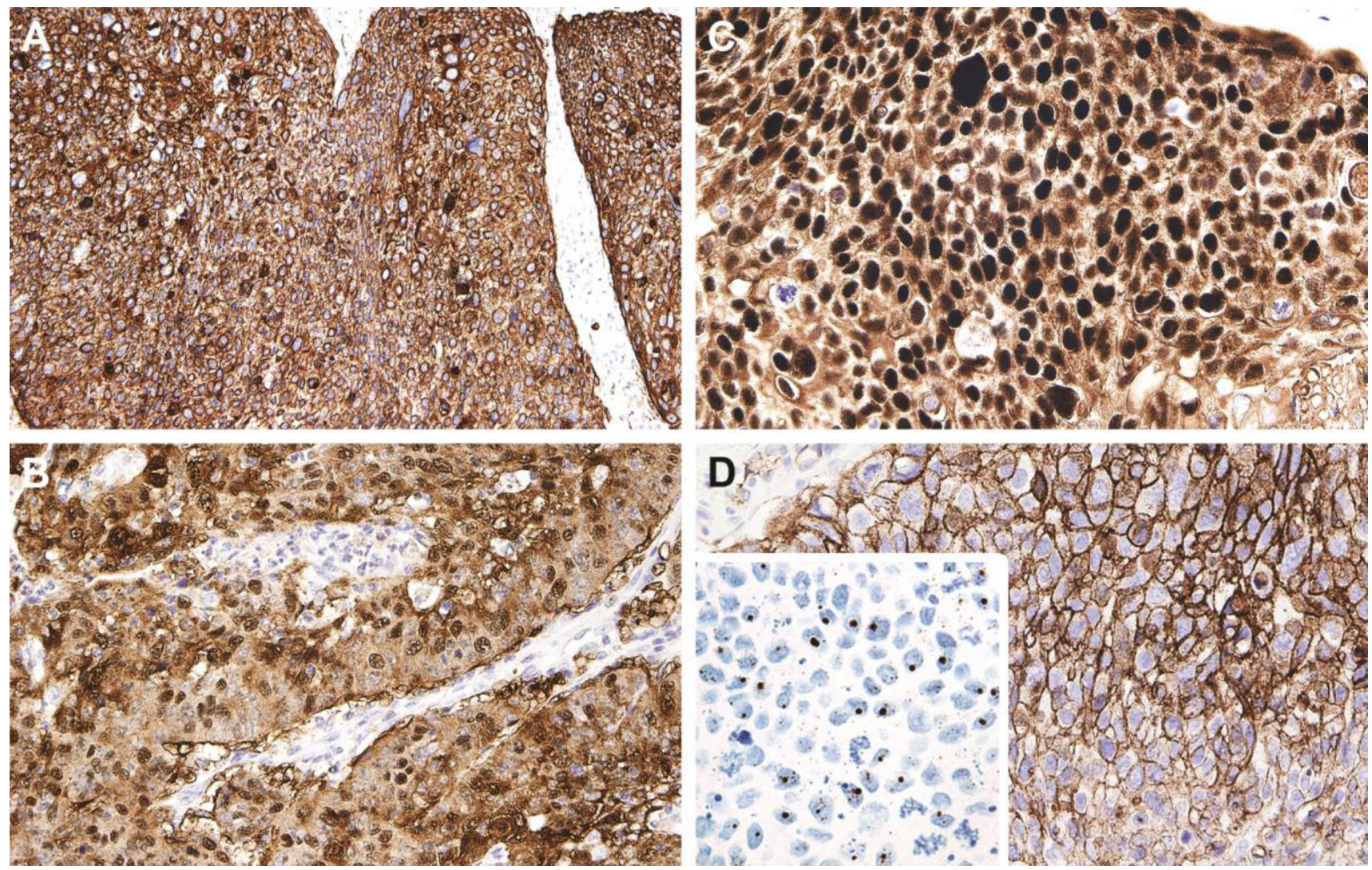

Fig. 5. Immunohistochemical features of urethral carcinoma

(A) Strong positivity for KRT5/6 in tumor cells. (B) Strong and diffuse staining for P16 in tumor cells. (C) Strong nuclear staining for P63 in tumor cells. (D) Strong membranous staining for thrombmodulin in tumor cells. Inset, Positive RNA hybridization signal for HPV HR7 (RNA scope analysis) in tumor cells. (A-D: DAB and hematoxylin; A-D and inset, $\times 200)$. 
A

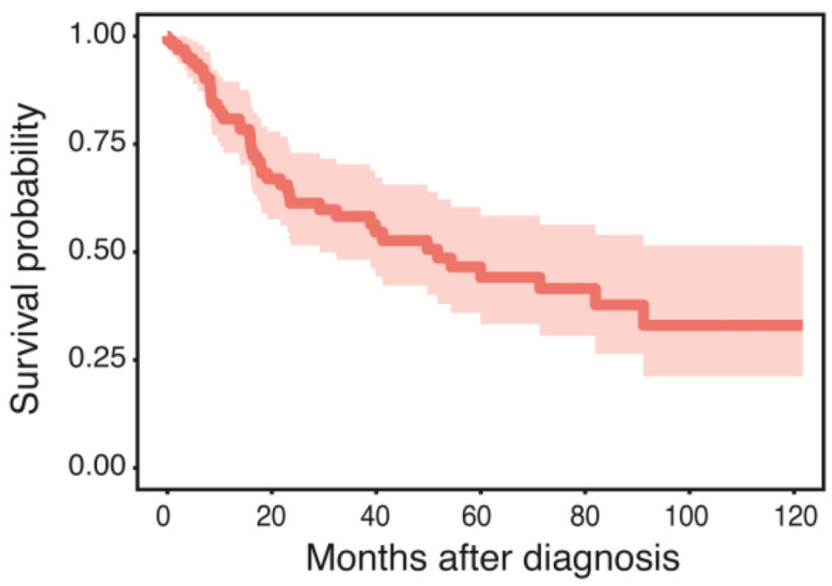

C

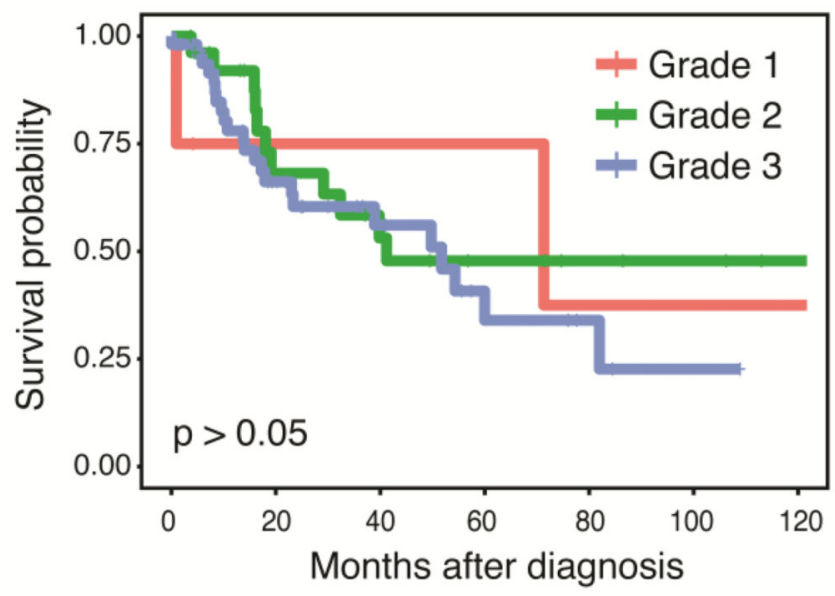

B

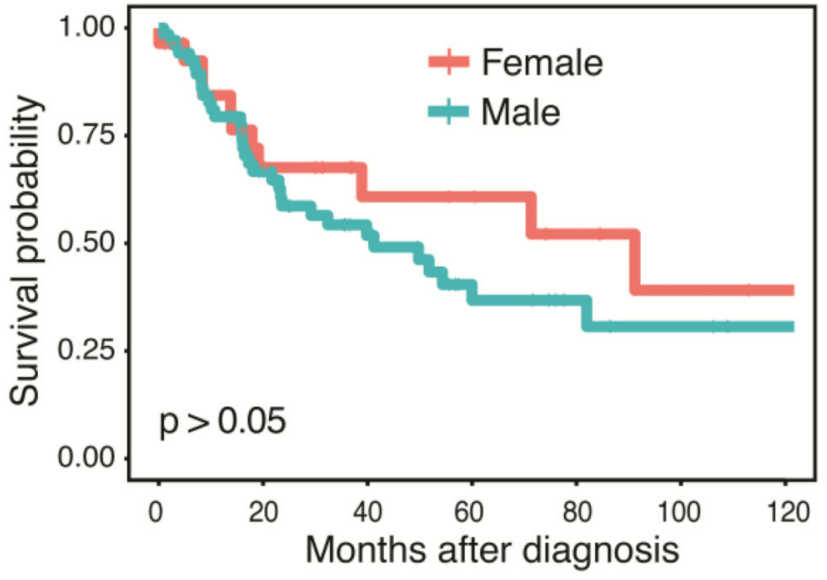

D

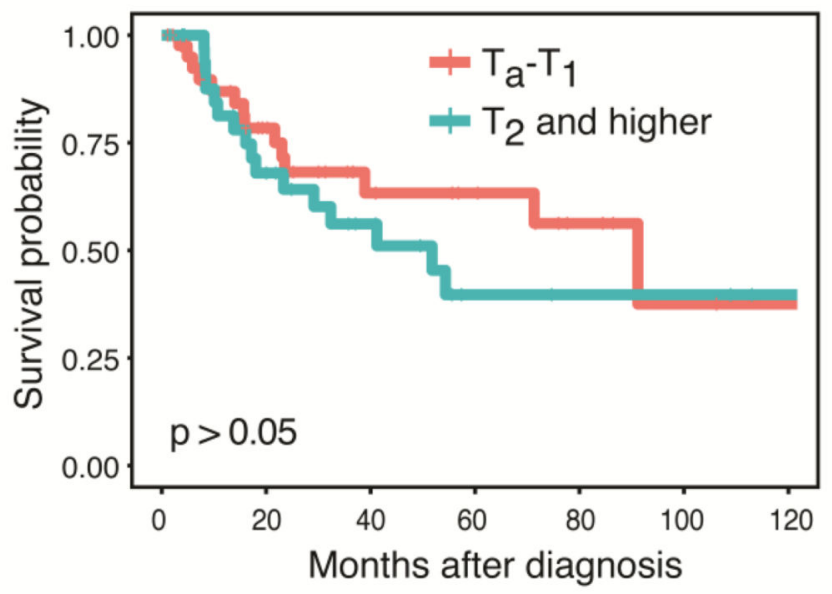

Fig. 6. Kaplan Meier survival analyses of patients with urethral carcinoma

(A) Overall survival analysis. Shaded area indicates the confidence interval. (B) Survival by gender. (C) Survival by histologic grade. (D) Survival by stage. 


\section{Table 1}

Summary of Clinical and Pathological Data of 106 Primary Urethral Carcinomas

\begin{tabular}{lll}
\hline Males & $73^{*}$ & $69.0+/-12.3 \mathrm{SD}$ years \\
Females & 33 & $72.8+/-14.6 \mathrm{SD}$ years \\
Total & 106 & $69.4+/-12.7 \mathrm{SD}$ years \\
\hline
\end{tabular}

Lymph node Metastases

\begin{tabular}{ll}
\hline Left inguinal & 13 \\
Right inguinal & 8 \\
Pelvic & 6 \\
Total & $\mathbf{2 7}$ \\
\hline
\end{tabular}

Distant Metastases

\begin{tabular}{ll}
\hline Lung & 7 \\
Liver & 3 \\
Adrenal & 1 \\
Bone & 3 \\
Pelvic soft tissue & 2 \\
Total & $\mathbf{1 6}$ \\
\hline Survival & \\
\hline With disease & $25 / 95 *(26.3 \%)$ \\
Without disease & $23 / 95(24.2 \%)$ \\
Median survival & 21 months \\
Mean survival & 39 months \\
\hline Immunohistochemistry \\
\hline KRT5/6 & $54 / 62 * *(87.1 \%)$ \\
KRT7 & $41 / 62(66.1 \%)$ \\
KRT20 & $6 / 62(9.6 \%)$ \\
P63 & $50 / 62(80.6 \%)$ \\
P16 & $44 / 70(62.8 \%)$ \\
Uroplakin & $0 / 50(0.00 \%)$ \\
Thrombomodulin & $51 / 62(82.2 \%)$ \\
GATA3 & $3 / 77(3.9 \%)$ \\
HPV18 & $7 / 19(36.8 \%)$ \\
\hline Number of cases/total number of cases with available data \\
\end{tabular}

